\title{
Las infraestructuras de telecomunicaciones en la Comunitat Valenciana
}

\author{
Narcís Cardona Marcet
}

Catedrático de la Universitat Politècnica de València y

Director de ITEAM (Instituto de Telecomunicaciones y Aplicaciones Multimedia

\section{Introducción: Telecomunicaciones, un bien esencial, antes, durante y después de la pandemia}

Las Telecomunicaciones han sido desde siempre uno de los pilares en los que se asienta el progreso de la Sociedad. Ya nadie duda de su importancia estratégica, y de que la carencia en redes e infraestructuras de comunicaciones deja atrás a aquellos países y regiones en los que su despliegue es escaso. Más recientemente hemos visto como las redes y servicios de telecomunicación han sido el soporte de las relaciones sociales y laborales durante la pandemia, y han resultado cruciales para mantener una buena parte de la actividad económica activa. Sin ellas la crisis del COVID habría sido aún más catastrófica.

Pero la pandemia ha impulsado en 2020 el uso de tecnologías de la información y comunicaciones en entornos públicos, privados y empresariales que, estando disponibles desde hace años, no se habían puesto en marcha o lo habían hecho muy tímidamente. Hablamos, entre otros, del teletrabajo, de la administración electrónica, de la telemedicina, o del comercio electrónico en toda su extensión. Todos ellos son servicios que, aún sin la pandemia como desencadenante, generan eficiencias, reducen costes y facilitan la vida de las personas.

Son las Tecnologías de la Información y las Comunicaciones (TIC), en definitiva, generadoras de sostenibilidad. En efecto, si reducimos el número de desplazamientos por carretera gracias al teletrabajo, usamos documentos electrónicos en lugar de papel, agilizamos algunas consultas médicas rutinarias con un buen sistema de atención remota, y completamos gestiones administrativas sin desplazamientos ni aglomeraciones en oficinas públicas, estamos evolucionando hacia una sociedad plenamente conectada y, precisamente por ello, sostenible.

Me permito pues desde estas líneas reclamar la importancia de la TIC en el progreso de nuestro país, y no retroceder en la oportunidad que nos ha dado la crisis del COVID para reactivar ese modelo de sociedad conectada que tanto puede aportar a la economía y al bienestar.

\section{Las Comunicaciones como derecho universal}

El acceso a telefonía básica, es decir, a línea telefónica de voz, es el único servicio que realmente se puede considerar que se mantiene como un derecho universal, por cuanto se garantiza la conectividad a toda la población, sea cual sea su lugar de 
residencia. Antaño, esta garantía se basaba en que era la empresa pública quien prestaba el servicio de telecomunicaciones. En España, la privatización de Telefónica, y la liberalización del mercado TIC, desarrollada en España entre 1998 y 2003 y no exenta de vaivenes políticos y geoestratégicos', incluyó el mantenimiento de carácter "universal" a la telefonía básica, pero no fue así para el resto de servicios de telecomunicaciones, por aquél entonces aún poco desarrollados, con excepción de las redes móviles GSM, que iniciaban una etapa de crecimiento exponencial.

La evolución del mercado y de la regulación, la privatización de servicios y las garantías necesarias para la libre competencia, han abocado a los servicios de telecomunicaciones a ser guiados por el negocio, olvidando progresivamente su carácter actual de bien esencial, de pilar de la cohesión y progreso social. Ya a finales de 1999, en el municipio granadino de Jun, se declaró el acceso a Internet como un derecho de toda su ciudadanía. Aquella osadía de su entonces alcalde fue pionera en Europa y en ese momento no transcendió a otros ámbitos.

Hoy, en 2021, los planes de banda ancha ${ }^{2}$ del Gobierno Nacional y Autonómico, que aplican desde 2013 las directrices de la Unión Europea sobre ayudas estatales para el despliegue rápido de redes de banda ancha (2013/C 25/01), contemplan como objetivo garantizar el acceso a internet para la población - para toda la población-, con anchos de banda ${ }^{3}$ que han ido creciendo desde los 10 a los 100 Megabits por segundo (Mbps).

Así, a diferencia de los que sucedía antes de la liberalización, quienes asumen las garantías de servicio a todos los núcleos de población son los gobiernos, en base principalmente a dichas ayudas públicas.

\subsection{El acceso universal de banda ancha, ¿una meta inalcanzable?}

El más reciente impulso a la banda ancha ${ }^{4}$ en España viene de la mano del Programa ÚNICO-Banda Ancha5 ${ }^{5}$ una actuación que se enmarca dentro de la Inversión 1 de la Componente 15 del Plan de Recuperación, Transformación y Resiliencia de la economía española y está financiado por la Unión Europea - NextGenerationEU, con fondos del Mecanismo de Recuperación y Resiliencia establecido por el Reglamento (UE) 2021/241 del Parlamento Europeo y del Consejo de 12 de febrero de 2021. El programa establece como objetivo conseguir que en la próxima década las infraestructuras de telecomunicaciones en España tengan la asequibilidad, disponibilidad, capacidad y resiliencia adecuadas para favorecer la capacidad económica de todas las personas y colectivos, y el desarrollo económico de cualquier zona geográfica, coadyuvando al reto demográfico. En concreto se especifica que

\footnotetext{
${ }^{1}$ Alabau, A. (2001), La Unión Europea y su política para la sociedad de la información. En el umbral de una nueva gobernanza europea. Fundación Airtel-Vodafone

2 PEBA-NGA: Programa de ayudas para la Extensión de la Banda Ancha de Nueva Generación (2013-2020); Secretaría de Estado para la Sociedad de la Información y la Agenda Digital; Órdenes IET/1144/2013; IET/241/2014; IET/275/2015 y ETU/275/2017

3 "Ancho de banda" en términos técnicos se refiere a la cantidad de espectro de frecuencias utilizado para una comunicación, aunque se ha aceptado su uso coloquial para referirse a la velocidad de transmisión de dicha conexión en bits por segundo.

${ }^{4}$ Téngase en cuenta que el término "banda ancha" se refiere en 2021 a velocidades de conexión por encima de $30 \mathrm{Mbps}$, aunque este mismo término se aplicaba años atrás a los $10 \mathrm{Mbps}, 2 \mathrm{Mbps}$, e incluso 256 kbps, lo cual es consecuencia de la evolución tecnológica y de la demanda de velocidades de transmisión cada vez mayores.

${ }^{5}$ Orden ETD/704/2021, de 25 de junio, por la que se modifica la Orden ETD/348/2020, de 13 de abril, por la que se establecen las bases reguladoras de la concesión de ayudas con cargo al Programa de extensión de la banda ancha de nueva generación; «BOE» núm. 157, de 2 de julio de 2021, páginas 79279 a 79288
} 
todos los hogares, rurales y urbanos, tengan gracias a este programa acceso a una conexión a Internet que ofrezca velocidades de descarga de al menos $100 \mathrm{Mbps}$.

Dichos planes se enfrentan a retos importantísimos, que trascienden a la mera distribución de ayudas, por cuantiosa que ésta pudiera ser. La tecnología con la que se despliega la banda ancha en ciudades no es necesariamente la mejor solución para las zonas rurales, ni para territorios con hábitats dispersos. Tampoco el despliegue de infraestructuras en zonas rurales tiene el mismo coste por kilómetro y habitante que en grandes núcleos de población, de modo que el reto que afronta España y Europa en este sentido es mucho mayor de lo que cabría imaginar.

Para entender a qué limitaciones y dificultades se enfrentan dichos planes, cabe revisar el histórico y las cifras actuales de despliegue de distintas tecnologías TIC. En la recopilación más reciente, publicada con datos agregados hasta 2020, se observa cómo el despliegue de redes de telecomunicaciones aparentemente alcanza cifras altas en nuestro país y en nuestra comunidad autónoma. Sin embargo, los indicadores que más se emplean en prensa y campañas de marketing se refieren a porcentajes de cobertura en número de habitantes con acceso a servicios de banda ancha, y no en número de municipios, viviendas o áreas -en el caso de telefonía móvil- cubiertas. Así, podemos encontrar fácilmente indicadores de cobertura del 99\% de la población en redes móviles 4G, o del 97\% de la población con acceso de banda ancha, o el 75\% con red de fibra óptica hasta el hogar. Si esos mismos indicadores lo referimos a municipios sin cobertura 4G, sin acceso de banda ancha o sin fibra óptica desplegada, el porcentaje baja sustancialmente. Este enfoque hasta cierto punto sesgado en la información, genera el espejismo de disponer de una red universal de comunicaciones de banda ancha y movilidad, que no es en absoluto real. Como ejemplo, la tabla siguiente muestra los indicadores de cobertura publicados por la Secretaría de Estado de Telecomunicaciones en mayo de $2021^{6}$, particularizados para la Comunitat Valenciana. Como es evidente, las cifras de cobertura de redes $3 \mathrm{C}$ o $4 \mathrm{C}$ corresponden al porcentaje de la población, y en apariencia indican que las redes móviles son accesibles desde cualquier punto del territorio, lo cual no es cierto si se representan los datos por número de municipios con cobertura 3G/4G, o áreas geográficas habitadas cubiertas por 3G/4G.

\begin{tabular}{|c|c|}
\hline Tecnología & Cobertura 2020 \\
\hline ADSL $\geq 2$ Mbps & $90 \%$ \\
\hline ADSL $\geq 10$ Mbps & $74 \%$ \\
\hline VDSL & $10 \%$ \\
\hline (WiFi) inalámbrica $\geq 30$ Mbps & $26 \%$ \\
\hline HFC & $67 \%$ \\
\hline FTTH & $86 \%$ \\
\hline (3G) UMTS con HSPA & $99,99 \%$ \\
\hline 4G (LTE) & $99,95 \%$ \\
\hline
\end{tabular}

Tabla 1. Cobertura en Comunitat Valenciana a 30 de junio de 2020 por tecnología

${ }^{6}$ Datos particularizados de cobertura de banda ancha en Comunitat Valenciana a 30 de junio de 2020; Publicado Mayo 2021, Secretaría de Estado de Telecomunicaciones e Infraestructuras Digitales. 


\section{Despliegue de redes de banda ancha}

\subsection{Despliegue de redes móviles, aparentemente el más extenso}

En el caso particular de telefonía móvil, se añade a las estadísticas mencionadas en el apartado anterior el sesgo creado por la definición de "cobertura", por comparación con la "conectividad". En términos generales, cobertura se refiere al área o áreas geográficas en las que es posible recibir señales de telefonía móvil. Sin embargo, dada la irregularidad de la propagación de ondas de radio, no es posible garantizar que todos los terminales de una misma área de cobertura sean capaces de conectarse de disponer de conectividad-al servicio móvil en cualquier punto de la misma. Por ese motivo, como indicador de la cobertura de servicio móvil se calcula el porcentaje de puntos con conectividad en una determinada área, o lo que es lo mismo, la probabilidad de que en un determinado punto se pueda acceder a la red móvil.

Por contra, en redes fijas - de acceso por cable o fibra óptica- sí se puede hacer referencia objetiva a la "conectividad", entendida como la disposición de una línea de conexión a Internet o telefonía fija. Así, la estadística que mide el despliegue de redes fijas lo hace en términos de puntos conectados, bien sean hogares, edificios, o municipios. En este caso, las cifras son objetivas, con las salvedades que se discuten en el apartado siguiente.

Los indicadores que publican, entre otros, la Secretaría de Estado de Telecomunicaciones e Infraestructuras Digitales, y anteriormente la Secretaría de Estado para el Avance Digital, en relación al despliegue de redes móviles en España y particularizados para la Comunitat Valenciana, corresponden al porcentaje de cobertura por municipios o, lo que es lo mismo, el tanto por ciento del área geográfica en la que se puede establecer conexión a la red móvil en los mismos. Comparando informes de años sucesivos, se puede observar como el despliegue de redes 4G (LTE) llegó muy rápidamente a los grandes núcleos de población y se ha extendido en 5 años a la práctica totalidad de los municipios de la Comunitat. Sin embargo, la interpretación de estos estadísticos debe hacerse con cautela. En el mapa siguiente, que compara la situación en 2016 y 2020, aunque en apariencia el despliegue de LTE se ha completado, en realidad solo se puede garantizar que se disponga de acceso a servicios de banda ancha móvil 4G en las zonas de color marrón oscuro. 


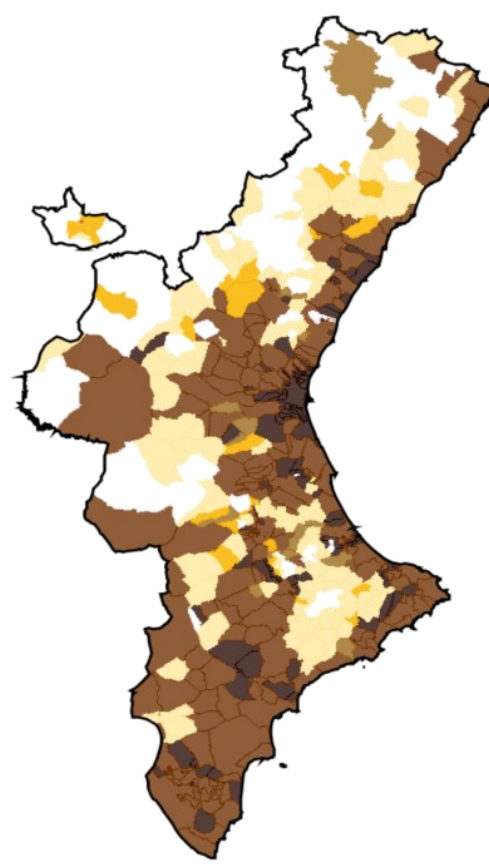

2016

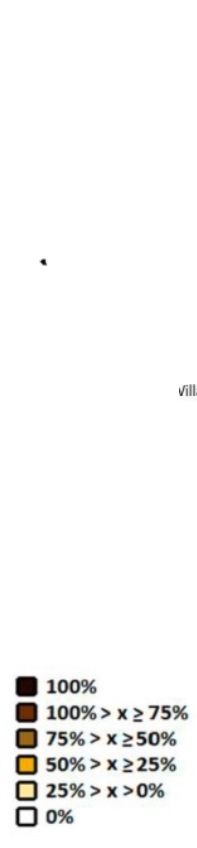

口 $0 \%$

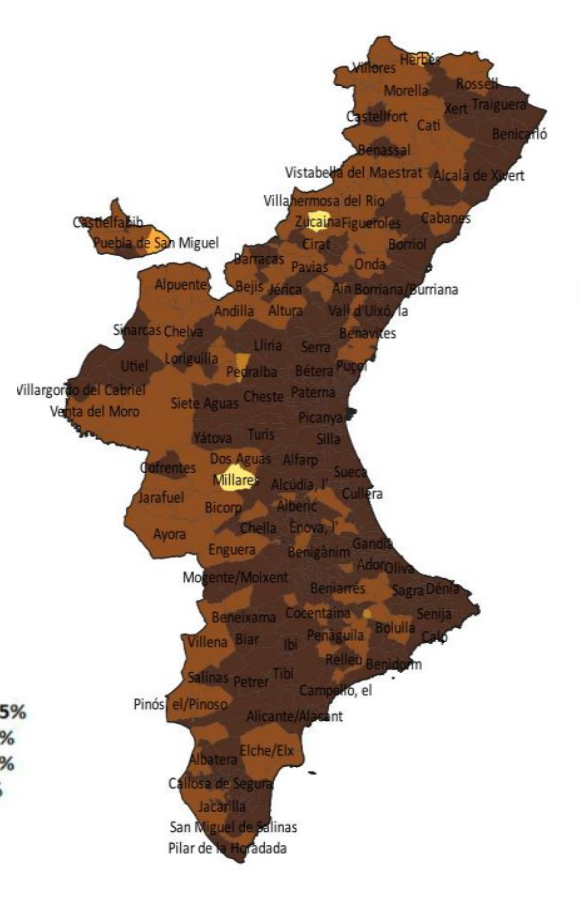

2020

Figura 7. Cobertura de LTE (4G) en la Comunitat Valenciana en porcentaje de área cubierta por municipio en 2016 y 2020. Fuente: Mayo 2021, Secretaría de Estado de Telecomunicaciones e Infraestructuras Digitales y Mayo 2016, Secretaría de Estado de Telecomunicaciones y para la Sociedad de la Información

Del mismo modo, se suelen presentar los datos agregados por porcentajes de cobertura, es decir, por el tanto por ciento del área del municipio que tiene conectividad a una determinada tecnología. En la tabla siguiente se reproduce la información publicada para la cobertura $4 \mathrm{G}$ en la Comunitat Valenciana, por número de hogares.

\begin{tabular}{|r|r|r|r|r|}
\hline $\begin{array}{l}\text { Rango de } \\
\text { cobertura }\end{array}$ & No Entidades & $\begin{array}{c}\text { No de habitantes } \\
\text { totales }\end{array}$ & No hogares totales & $\begin{array}{c}\text { Hogares cubiertos } \\
\text { LTE }\end{array}$ \\
\hline $90 \leq x \leq 100$ & 1.090 & 4.999 .393 & 1.981 .133 & 1.980 .919 \\
\hline $80 \leq x<90$ & 21 & 1.483 & 607 & 524 \\
\hline $70 \leq x<80$ & 14 & 488 & 203 & 153 \\
\hline $60 \leq x<70$ & 9 & 137 & 54 & 35 \\
\hline $50 \leq x<60$ & 2 & 754 & 309 & 176 \\
\hline $40 \leq x<50$ & 4 & 937 & 387 & 186 \\
\hline $30 \leq x<40$ & - & - & - & -19 \\
\hline $20 \leq x<30$ & 4 & 193 & 85 & 0 \\
\hline $10 \leq x<20$ & 1 & 2 & 1 & 4 \\
\hline $0 \leq x<10$ & 45 & 382 & 171 & 1.982 .949 \\
\hline Totales & 1.190 & 5.003 .769 & & 1.982 .019 \\
\hline
\end{tabular}

Tabla 2. Rango de cobertura LTE (4G) en Comunitat Valenciana a 30 de junio de 2020 por entidades singulares 
La interpretación correcta de la tabla debería hacerse teniendo en cuenta los porcentajes de la primera columna, y con ello el número efectivo de hogares no cubiertos por LTE, que en apariencia es pequeño, se acerca en realidad a los 100.000, un $5 \%$ del total en la Comunitat Valenciana.

\subsection{Despliegue de redes fijas. Un coste mayor para conexiones garantizadas}

El despliegue de redes fijas de banda ancha es en este momento mucho menos extenso que el de redes móviles, ya que en éstas no es necesario llegar a todos los hogares en base a una canalización de cable o fibra óptica, sino mediante acceso radio, inalámbrico. La comparación de los datos de cobertura $4 \mathrm{G}$, de los que se deduce que el 95\% de los hogares dispone de acceso a esta tecnología, con los de la cobertura de banda ancha - más de 30Mbps- proporcionada por cualquier tipo de red fija, refleja la mayor dificultad en el despliegue de acceso por cable o fibra, y el riesgo que supone en el aumento progresivo de la brecha digital entre zonas urbanas y rurales. En efecto, los datos de la tabla 4 indican que hasta un $8 \%$ de los hogares de la Comunitat Valenciana no tiene acceso a banda ancha (30 Mbps).

\begin{tabular}{|r|r|r|r|r|}
\hline $\begin{array}{l}\text { Rango de } \\
\text { cobertura }\end{array}$ & No Entidades & $\begin{array}{c}\text { N2 de habitantes } \\
\text { totales }\end{array}$ & No hogares totales & $\begin{array}{c}\text { Hogares cubiertos } \geq \\
\text { 30 Mbps }\end{array}$ \\
\hline $90 \leq x \leq 100$ & 777 & 4.504 .120 & 1.784 .658 & 1.759 .853 \\
\hline $80 \leq x<90$ & 66 & 232.035 & 90.932 & 77.722 \\
\hline $70 \leq x<80$ & 38 & 101.704 & 40.756 & 30.051 \\
\hline $60 \leq x<70$ & 12 & 30.383 & 11.648 & 7.689 \\
\hline $50 \leq x<60$ & 8 & 6.580 & 2.724 & 1.539 \\
\hline $40 \leq x<50$ & 13 & 109.005 & 44.183 & 20.813 \\
\hline $30 \leq x<40$ & 4 & 303 & 126 & 42 \\
\hline $20 \leq x<30$ & 9 & 9.934 & 3.835 & 1.031 \\
\hline $10 \leq x<20$ & 9 & 719 & 303 & 40 \\
\hline $0 \leq x<10$ & 254 & 8.986 & 3.782 & 10 \\
\hline Totales & 1.190 & 5.003 .769 & 1.982 .949 & 1.898 .791 \\
\hline
\end{tabular}

Tabla 3. Distribución de las entidades singulares por rango de cobertura de redes a velocidades $\geq 30$ Mbps para la prestación de servicios de banda ancha desde una ubicación fija a 30 de junio de 2020 en Comunitat Valenciana

El informe antes referido, publicado por la Secretaría de Estado, también confirma que esta carencia se produce principalmente en zonas rurales o menos pobladas, como se puede apreciar en la figura y tablas comparativas siguientes. 
Cobertura $\geq 30$ Mbps por geotipos de población a nivel de municipio (2020) -

\section{Valenciana}

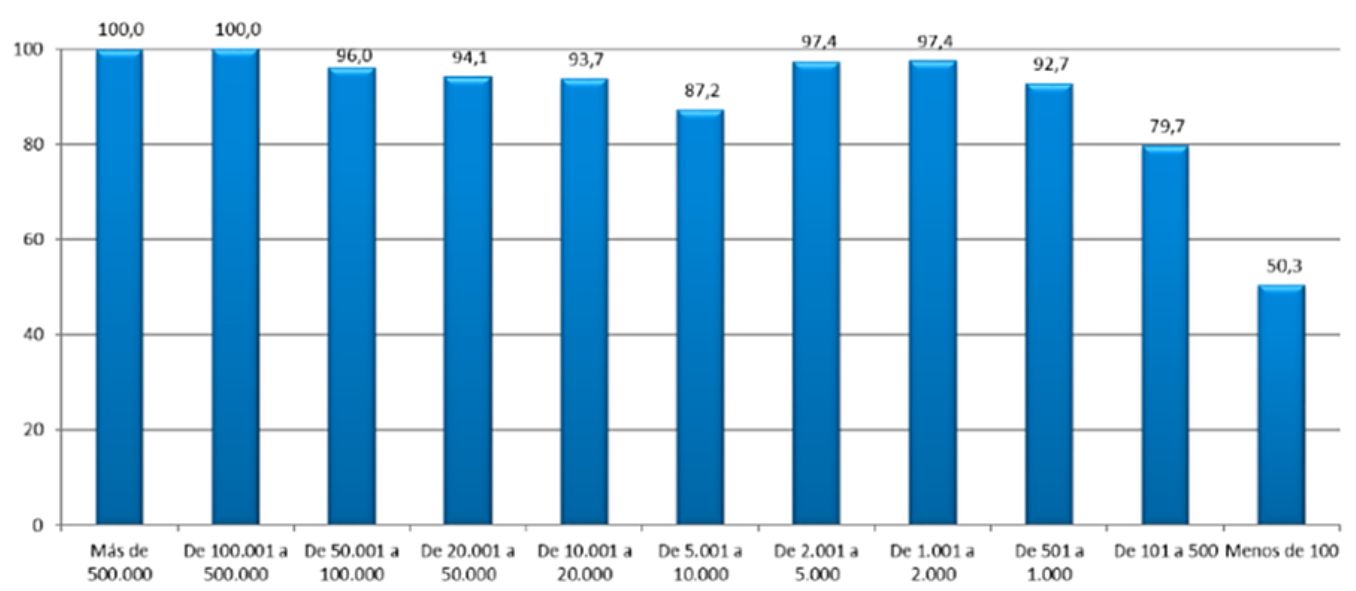

\% $\%$ cobertura de hogares

Cobertura $\geq 100$ Mbps por geotipos de población a nivel de municipio (2020) C. Valenciana

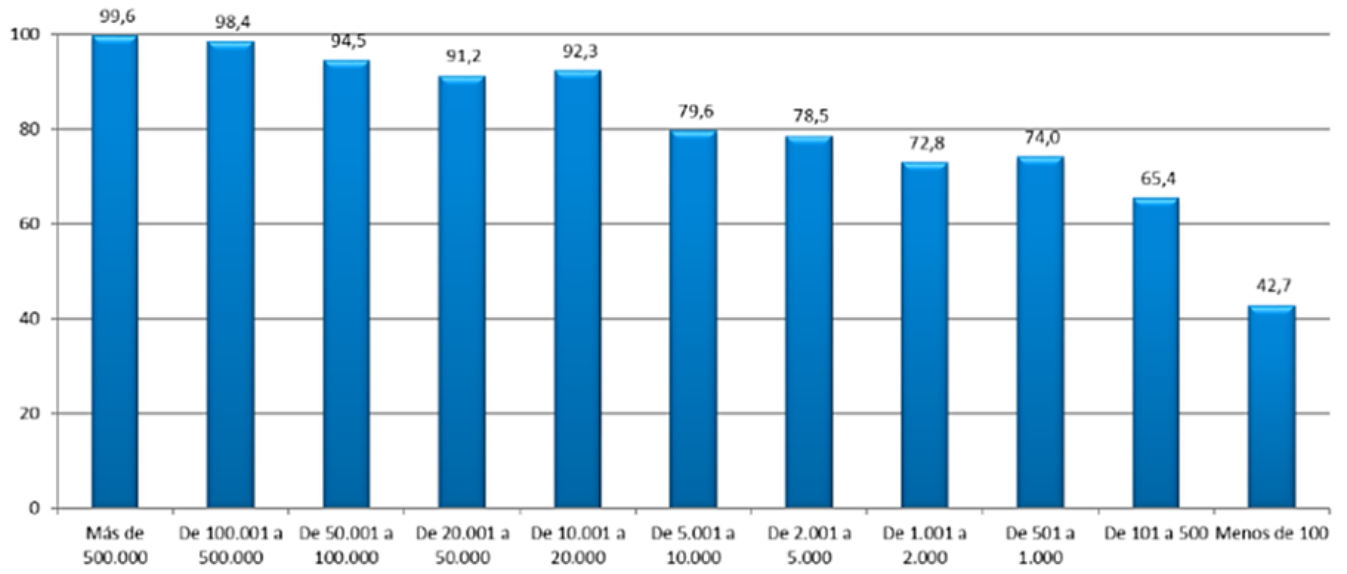

a $\%$ cobertura de hogares

Figura 3. Comparación entre la cobertura de redes a velocidades $\geq 30$ Mbps y a $\geq 100 \mathrm{Mbps}$ para la prestación de servicios de banda ancha desde una ubicación fija por geotipo de población a nivel de municipio en Comunitat Valenciana a 30 de junio de 2020

Sin embargo, no debemos dejar de mencionar que los datos comparados entre 2018 y 2020 demuestran que el despliegue de fibra óptica (FTTH) ha cubierto en el periodo de tres años amplias zonas con municipios de menos de 2000 habitantes a las que no llegaba en 2018. 


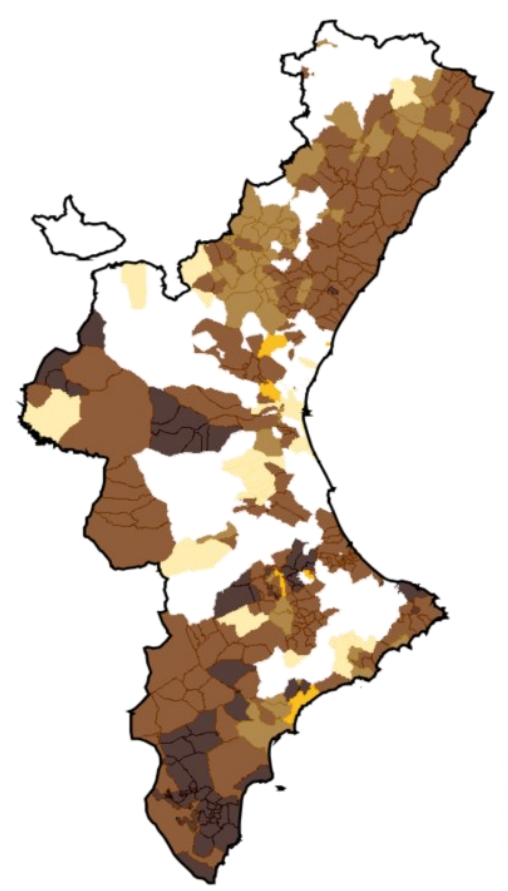

2018

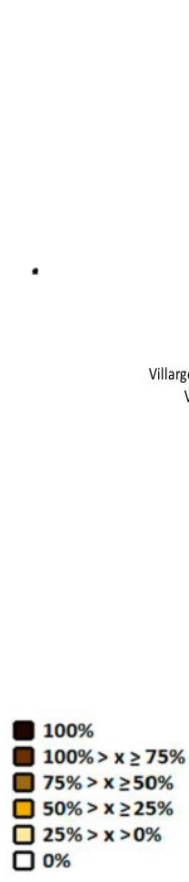

$0 \%$

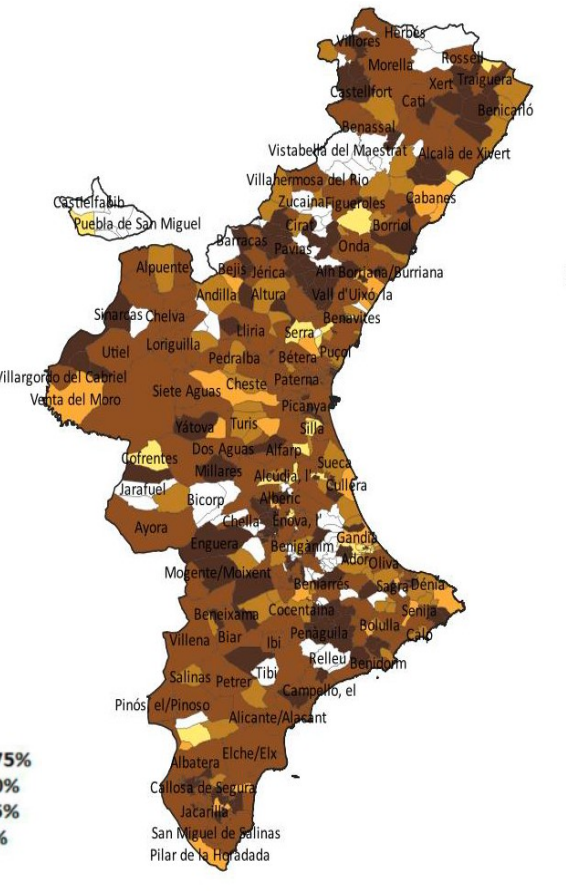

2020

Figura 2. Comparativa de la cobertura de banda ancha (>100Mbps) mediante redes fijas en la Comunitat Valenciana en porcentaje de área cubierta por municipio en 2020 y 2018. Fuente: Mayo 2021, Secretaría de Estado de Telecomunicaciones e Infraestructuras

\section{Digitales}

\subsection{Planes de banda ancha para reducir la brecha digital}

Los planes de banda ancha pretenden en parte suplir las deficiencias antes citadas y mirar a los despliegues de las redes desde el punto de vista de los municipios no cubiertos, de la población que no tiene acceso a redes y servicios de banda ancha, y de los territorios a los que la falta de rentabilidad comercial de los despliegues ha ido dejando atrás en el rápido progreso de las TIC.

Recuperar la actividad en zonas menos pobladas pasa necesariamente por recuperar su conectividad. Si hace un siglo la construcción de carreteras y vías de ferrocarril era la clave del progreso, hoy también lo es el despliegue de infraestructuras de telecomunicaciones, ayudando a vertebrar y cohesionar los territorios y manteniendo a las zonas rurales activas, vivas, plenamente conectadas.

Para ello, el programa UNICO identifica como "zonas blancas" a aquéllas que no disponen de cobertura de redes de velocidad de al menos $30 \mathrm{Mbps}$, ni planes para su dotación en los próximos tres años, y como "zonas grises" a las que, aun teniendo cobertura o previsiones de cobertura de muy alta velocidad en los próximos tres años, es proporcionada por un solo operador y la velocidad es inferior a $100 \mathrm{Mbps}$. Esto totaliza 258.146 zonas elegibles en España, que albergan 1.583.604 hogares y viviendas, obviamente su inmensa mayoría ubicadas en núcleos de población pequeños y zonas rurales. 
En la Comunitat Valenciana se han identificado 37.648 zonas blancas y 7.041 zonas grises, que corresponden a más del $17 \%$ del total nacional, distribuidas entre las tres provincias como muestra el cuadro siguiente

\begin{tabular}{|l|r|r|r|r|r|r|}
\hline \multicolumn{1}{|c|}{ Provincia } & \multicolumn{1}{|c|}{$\begin{array}{c}\text { No Zonas } \\
\text { Blanca NGA }\end{array}$} & $\begin{array}{c}\text { No Zonas } \\
\text { Gris NGA }\end{array}$ & $\begin{array}{c}\text { Total } \\
\text { Zonas }\end{array}$ & $\begin{array}{c}\text { Unidades } \\
\text { Inmobiliarias } \\
\text { Blancas }\end{array}$ & $\begin{array}{c}\text { Unidades } \\
\text { Inmobiliarias } \\
\text { Grises }\end{array}$ & $\begin{array}{c}\text { Total } \\
\text { Unidades } \\
\text { Inmobiliarias }\end{array}$ \\
\hline Alicante/Alacant & 11.628 & 6.346 & 17.974 & 42.058 & 23650 & 65.708 \\
\hline Castellón/Castelló & 8.175 & 69 & 8.244 & 33.326 & 378 & 33.704 \\
\hline Valencia/València & 17.845 & 626 & 18.471 & 78.705 & 3791 & 82.496 \\
\hline Total Com. Valenciana & 37.648 & 7.041 & 44.689 & 154.089 & 27.819 & 181.908 \\
\hline Total (España) & $\mathbf{2 2 2 . 6 9 1}$ & $\mathbf{3 5 . 4 5 5}$ & $\mathbf{2 5 8 . 1 4 6}$ & $\mathbf{1 . 3 2 6 . 3 4 9}$ & $\mathbf{2 5 7 . 2 5 5}$ & $\mathbf{1 . 5 8 3 . 6 0 4}$ \\
\hline
\end{tabular}

Tabla 4. Distribución de zonas blancas y grises identificadas en el programa UNICO en la Comunitat Valenciana. Fuente: Ministerio de Asuntos Económicos y Transformación Digital.

\section{La fibra óptica como esqueleto imprescindible de la banda ancha}

Es ya bien conocido que los despliegues de fibra óptica son imprescindibles para alcanzar el acceso a banda ancha con garantías de conectividad. Se ha publicado y anunciado con reiteración que el despliegue de fibra óptica en España es el mayor de Europa, con cifras de kilómetros de fibra desplegados por encima de la suma de los de tres países más desarrollados del continente: Alemania, Francia y Reino Unido. Siendo las cifras ciertas, es relevante matizar que la inmensa mayoría de los despliegues citados corresponden a la conectividad de hogares, y en núcleos de población grandes, ya que están orientados a la banda ancha doméstica. Por el tipo de tecnología que usa la conectividad por fibra óptica, ésta puede garantizar velocidades de acceso a Internet cerca de 10 veces superiores a las de las redes móviles y a otras tecnologías por cable. En esa magnitud podemos cifra también la brecha digital entre zonas urbanas con despliegues de fibra y zonas, no solo rurales sino también industriales, donde el despliegue de fibra es más caro por kilómetro y usuario, y donde por tanto no se priorizan dichos despliegues por parte de los grandes operadores.

Son numerosas las empresas que han proliferado como operadores locales en las citadas zonas, y en muchos casos son éstas las que han permitido a muchos municipios de nuestro país disponer de acceso de banda ancha, y en ocasiones incluso de acceso básico a Internet. La combinación de despliegues de operadores nacionales y locales con la ayuda que los planes de banda ancha pueden prestar, con ayuda de las administraciones locales y autonómicas, debería frenar el crecimiento de la brecha digital entre el mundo rural y urbano en materia de conectividad TIC.

En este punto cabe hacer mención a tres aspectos de los despliegues de fibra óptica que pasan más desapercibidos en los medios de comunicación.

El primero de ellos se refiere a la canalización, mejor dicho, la "no canalización" de la red en el último kilómetro, desde la cabecera de red hasta el hogar. En muchas 
ocasiones, tanto en ciudades como en pequeñas poblaciones, los cables de comunicaciones siguen recorriendo de forma muchas veces caótica las fachadas de los edificios. En el caso de los edificios de nueva construcción este aspecto se evita al aplicar en todos ellos la normativa de Infraestructuras Comunes de Telecomunicaciones (ICT) que obliga a canalizar los cables de comunicaciones y televisión por el interior de los edificios. Sin embargo, el parque de edificios y viviendas anterior a la aplicación de las normas de ICT no está obligado a una canalización que, de no realizarse en obra, es muy costosa para los particulares y las administraciones. La solución actual es la tirada por fachada de los cables de comunicaciones, la cual merece una revisión de las normas ICT o una regulación a cualquier nivel que reduzca el impacto visual de estos despliegues.

En segundo lugar, es también poco conocido que la fibra óptica es imprescindible para las comunicaciones móviles, es decir, para el despliegue de redes $4 \mathrm{C}$ y, mucho más, en las nuevas redes 5G. Los anchos de banda (velocidades de transmisión) que circulan por las redes móviles solo pueden canalizarse desde las antenas hacia el resto de la red mediante enlaces de muy gran ancho de banda, que solo la fibra óptica puede proporcionar en casi todos los escenarios. De la "fibra hasta el hogar" citada en el apartado anterior, pasmos al concepto de "fibra hasta la antena", que nos deja ver de forma muy esquemática las rede móviles 5G como un esqueleto de fibra óptica de cuyas ramificaciones se conectan antenas para acceso móvil.

Finalmente, si bien la fibra óptica es mucho mejor en velocidad y fiabilidad que otras alternativas, también es cierto que no es posible hacerla llegar a cualquier ubicación. El despliegue de fibra hasta poblaciones muy aisladas o en hábitats muy dispersos no es viable económicamente $y$, aún financiado con ayudas estatales, no lo es en ocasiones por limitaciones técnicas o medioambientales. En esos casos la solución, evidentemente, es llegar sin cables, mediante conexión vía satélite, mediante la red móvil, o a través de acceso inalámbrico punto a multipunto, y siempre a costa de conseguir menos velocidad de acceso.

\section{La 5G, ¿por la misma senda que la 46 ?}

La quinta generación de redes móviles se ha concebido para superar a sus predecesoras en muchos aspectos, empezando por la velocidad de acceso, por el ancho de banda. Realmente para la mayoría de servicios a los que los usuarios de telefonía móvil acceden a diario, no sería estrictamente necesario dar el salto de $4 \mathrm{C}$ a 5G, ya que no consumen tanto ancho de banda como para llegar al límite por conexión de las redes actuales. Para la tecnología 4G podríamos estimar en un techo máximo de $100 \mathrm{Mbps}$ por conexión, pero que se reduce a unos $20 \mathrm{Mbps}$ (de descarga) en buenas condiciones de conexión en redes comerciales.

56 crece respecto a las generaciones anteriores "horizontalmente", es decir, no solo aporta más ancho de banda, sino que abarca un mayor número de usuarios y equipos que se pueden conectar a la red, en casi cualquier banda de frecuencias y en muchos escenarios para los cuales no se diseñaron las generaciones previas, enfocadas casi exclusivamente a terminales móviles de bolsillo.

En cuanto al despliegue de acceso a Internet mediante redes 5G, es decir, al despliegue de la cobertura 5G, la tendencia natural es la misma que en sus predecesoras: cubrir en una primera fase los núcleos de población y escenarios donde 
hay más concentración de usuarios, y progresivamente ir abarcando otras áreas menos densas. Esto dejaría de nuevo los planes de cobertura de zonas rurales para el final del ciclo de despliegue, al menos 5 años después de las primeras instalaciones. Sin embargo, y dado que $5 G$ se puede desplegar en muchas bandas de frecuencias posibles, se ha licitado la banda de 700 megahercios $(\mathrm{MHz})$ recientemente, con el fin primario de facilitar el despliegue de $5 \mathrm{C}$ en zonas suburbanas y rurales. En efecto, las frecuencias de $700 \mathrm{MHz}$, antes utilizadas por la radiodifusión de televisión, tienen más alcance y permiten cubrir distancias mayores que las utilizadas hasta la fecha. Esta facilidad de propagación las hace idóneas para zonas rurales, donde la población está más dispersa y por tanto requiere de antenas con coberturas (alcances) mayores. En este punto cabe destacar que la banda de $700 \mathrm{MHz}$ no es la solución universal para la "banda ancha rural", ya que como contrapartida a su largo alcance no puede desarrollar anchos de banda tan grandes como sucede en $3.5 \mathrm{GHz}$, actualmente ya usada en despliegue $5 \mathrm{G}$ en ciudades. En este caso, la brecha en el uso de $700 \mathrm{MHz}$ puede suponer unas 3 o 4 veces menor ancho de banda que en $3.5 \mathrm{GHz}$, alcanzando a lo sumo unos $30 \mathrm{Mbps}$, no muy lejos de los valores de $4 \mathrm{G}$.

No siendo tan competitiva $5 \mathrm{G}$ en entornos rurales, y no siendo imprescindible alcanzar los anchos de banda de 5C para la mayoría de servicios en terminales de bolsillo, ¿dónde destaca entonces esta nueva generación de redes móviles? La 50 ha sido diseñada -también, y a diferencia de sus predecesoras- para la conectividad de máquinas y de forma masiva, y éstas imponen unos requisitos de inmediatez en la transmisión (latencia), de ancho de banda y de consumo de energía que no era posible alcanzar con 4G. Es en el sector industrial donde 50 tiene un papel muy importante que jugar $y$, por eso es muy importante que la fibra óptica, el esqueleto sobre el que se sustenta el despliegue de 5G, llegue a los polígonos industriales y a los centros de producción, logísticos y portuarios.

La combinación de un despliegue estratégico de fibra óptica y de la aplicación de 50 en los entornos industriales, puede hacer muy competitiva a nuestra industria.

De nuevo, y como ya hemos visto que sucede con los despliegues en zonas rurales, los modelos de negocio basados en conectividad, en líneas de datos, no son tan rentables para el operador en este momento y puede que no lo sean a medio plazo hasta que el número de dispositivos a conectar en entornos industriales crezca sustancialmente. Esto hace que la gran oportunidad que representa $5 \mathrm{G}$ para nuestra industria se pueda desplazar más de 5 años a futuro y, por ello, perderse. Y de nuevo tal como sucede con los despliegues de redes de cable y fibra en zonas rurales, deberían ser operadores locales quienes cubrieran esos nichos de despliegue que a medio plazo no son rentables para los grandes operadores nacionales. No obstante, no existe esa posibilidad en redes móviles, dado que la regulación actual autoriza el uso del espectro de frecuencias para servicio móvil por licitación exclusiva en todo el territorio. Es importante, y ya se está empezando a discutir esta posibilidad, que se liciten frecuencias de telefonía móvil para uso privativo, para pequeños operadores locales o para grandes empresas que, sin menoscabo de las leyes de competencia y sin perjudicar a los operadores actuales, puedan desarrollar y operar redes -privadas o públicas- $5 \mathrm{G}$. 


\section{Conclusiones. Planificar el futuro activando el presente.}

Siendo las Telecomunicaciones un bien esencial para la sociedad actual, y habiendo demostrado su fortaleza como tecnología, y su verdadero potencial como elemento vertebrador de muchas de las actividades económicas de nuestro país en una situación tan compleja como la pandemia de 2020, no es aventurado asegurar que deberían considerarse un bien de interés público en toda su extensión. De alguna forma este concepto es la base los planes de banda ancha actuales, tanto de la Comisión Europa como del Gobierno de España, que, de llevarse a cabo con éxito, deberían servir para evitar la brecha digital que constantemente afecta a las zonas menos pobladas, y asegurar la plena conectividad de todos los territorios.

La Comunitat Valenciana, como el resto de España, destaca en Europa en despliegue de fibra óptica, en implantación de servicios de Telecomunicaciones y en la puesta en marcha de 5C. Sin embargo, debemos acelerar la digitalización de la industria y la conectividad rural. Lo contrario supondrá una pérdida de oportunidades difícilmente reparable, tanto en la competitividad industrial como en la vertebración del territorio.

Los datos estadísticos de acceso a servicios de banda ancha en nuestra Comunitat, bien sea por fibra, por móvil (4G) o por otras tecnologías por cable y radio, siguen indicando que un porcentaje significativo de zonas rurales o no densamente pobladas no tienen acceso de banda ancha (a 10Mbps), en contraste con núcleos de población grandes, donde se superan fácilmente los $100 \mathrm{Mbps}$. Este factor 10 en capacidad de conexión genera desequilibrios y aumenta el sesgo demográfico.

Ni la fibra óptica ni el 5G por sí mismos son suficientes para llegar a garantizar la banda ancha en toda la geografía Valenciana, ni siquiera en combinación con otras tecnologías por satélite o terrestres, pero es necesario facilitar el despliegue de fibra y dotar de competencia a operadores locales para desplegar acceso 5G, y llegar cuanto antes a cifras elevadas de conectividad rural y empresarial. No es necesario crear tecnología nueva para cubrir esa brecha, ya está disponible, solo hay que activarla, impulsar su despliegue y ponerla en marcha allí donde puede generar más riqueza e igualdad de oportunidades,

A mayor conectividad, mayor competitividad, igualdad y sostenibilidad.

\section{Bibliografía:}

ALABAU Muñoz, Antonio, 2001. La Unión Europea y su política para la sociedad de la información. En

el umbral de una nueva gobernanza europea. Editorial Fundación Vodafone. ISBN: 978-8493029883

Ministerio de Industria, Turismo y Comercio, 2009. Informe Final del Programa de Extensión de Banda Ancha (PEBA). Disponible en: https://avancedigital.gob.es

PEBA-NGA: Programa de ayudas para la Extensión de la Banda Ancha de Nueva Generación (2013-2020); Secretaría de Estado para la Sociedad de la Información y la Agenda Digital; Órdenes IET/1144/2013; IET/241/2014; IET/275/2015 y ETU/275/2017.

Disponible en https://portalayudas.mineco.gob.es/banda-ancha/Paginas/Index.aspx 
Comisión Nacional de los Mercados y la Competencia. 2020. IAP/CNMC/001/20: INFORME DE LA CNMC SOBRE LAS AYUDAS PÚBLICAS EN ESPAÑA. Disponible en: https://www.cnmc.es/expedientes/iapcnmc00120

Observatorio nacional de las telecomunicaciones y de la Sociedad de la Información; Red.Es; 2020. La sociedad en red; transformación digital en España. Informe anual 2019. Edición 2020. Disponible en: http://ontsi.red.es

Orden ETD/704/2021, de 25 de junio, por la que se modifica la Orden ETD/348/2020, de 13 de abril, por la que se establecen las bases reguladoras de la concesión de ayudas con cargo al Programa de extensión de la banda ancha de nueva generación; «BOE» núm. 157, de 2 de julio de 2021, páginas 79279 a 79288

Secretaría de Estado de Telecomunicaciones e Infraestructuras Digitales. Mayo 2021. Datos particularizados de cobertura de banda ancha en Comunitat Valenciana a 30 de junio de 2020. Disponible en: https://avancedigital.mineco.gob.es 